This document is a preprint of an article accepted for publication by Current Opinion in Behavioral Sciences.

DOI for the published journal article will be provided when obtained

(C) 2021. This manuscript version is made available under the CC-BY-NC-ND 4.0 license https://creativecommons.org/licenses/by-nc-nd/4.0/ 


\title{
Knismesis: the aversive facet of tickle
}

\author{
Anton A. Varlamov ${ }^{\mathrm{a}, \mathrm{b} *}$, Ivan V. Skorokhodov ${ }^{\mathrm{a}, \mathrm{b}}$ \\ ${ }^{\text {a }}$ Cognition and Communication Laboratory, Pushkin State Russian Language Institute, Moscow, Russia. 6 \\ Ac. Volgin Str., Moscow, 117485, Russia \\ ${ }^{b}$ ASD Rehabilitation Center "Our Sunny World", Moscow, Russia. 11A Gorlov tupik, Moscow, 127055, \\ Russia \\ * corresponding author \\ antonvarlamov@gmail.com
}

Cognition and Communication Laboratory, Pushkin State Russian Language Institute. 6 Ac. Volgin Str., Moscow, 117485, Russia

\section{Abstract}

There are two different kinds of tickle, knismesis (feather-light tickle) and gargalesis (more intense tickle eliciting involuntary laughter). In this article we review earlier and recent advances in tickle psychophysics and in neurophysiology of touch afferents and hypothesize that knismesis is signaled by populations of rapidly adapted hair follicle afferents, can stimulate itch neurons via dorsal horn projections, and is heavily moderated by afferent inputs from other touch neurons. Finally, we suggest that pathological light touch intolerance observed in autism spectrum disorder may reflect knismesis hyper-responsiveness and attenuated knismesis inhibition stemming from impaired integration of sensory inputs from other touch submodalities.

\section{Introduction}

As early as 1897 Hall and Allin [1**] described tickle as comprised of two different sensations, gargalesis (more intense tickle eliciting involuntary laughter) and knismesis (generally unpleasant feather-light tickle). Up until now there is no single consensus definition of tickle, and most review articles prefer not to provide a single definition but, rather, to stress the two-faceted nature of tickle [2-5]. Both gargalesis and knismesis are arousing and urgent, and both invoke an orienting reaction generally followed by the behavior response aimed to cease the sensation. In most Indo-European languages including English, French, German, Russian, and Hindi there is a single word for tickle having two or more different meanings related to gargalesis and knismesis [6,7]; it suggests that despite the prominent difference between these sensations they are conceived as closely related.

Gargalesis is elicited by moderately intense repetitive stimulation of specific sensitive areas (soles of feet, armpits, ribs, neck), induces involuntary laughter, and is considered the more social kind of tickle associated with complex emotional and behavioral response and social play. The link between light touch and laughter has been observed in many animal species. When stroked in a similar manner, rats vocalize at a frequency of about $50 \mathrm{kHz}$; this sound is considered an equivalent of human laughter and is also emitted during social play $[8,9]$. Great apes, when tickled, laugh in a manner very similar to humans, and their tickle games are very reminiscent to human parent-child playful tickle interactions $\left[3^{* *}, 5,10\right]$. Another important feature of gargalesis is that you cannot elicit this sensation in self; this is attributed to the feeling of agency in self-produced touch and to attenuation of somatosensation via the efference copy $[2,11]$. Gargalesis is believed to play an important role in affiliative behavior, evolution of social play, humor, and speech, and in developing the distinction between self and others [2-5]. It should be noted that despite its relation to laughter gargalesis always elicits a withdrawal response and is not always related to pleasure. Many adults do not find gargalesis enjoyable, and there can easily be too much of 
gargalesis: if it is invoked forcefully, a playful interaction turns into a display of dominance or even to torture $[2-4 *]$.

Knismesis is produced by very light moving touch and elicits an arousing feeling often referred to as disquiet, slight discomfort, or 'spidery' sensations; it is often linked to itch. Unlike gargalesis, knismesis does not elicit laughter and you can effectively induce knismesis in yourself by moving a feather across your skin or by gently touching your body hairs. From the evolutionary perspective, the suggested role of knismesis is to alert us to blood-sucking insects and skin parasites [2-5]. Most studies of tickle have been focused primarily on gargalesis while knismesis is considered the simpler and less enigmatic variety of tickle; despite that, little is known about the physiological background of knismesis. Some behavioral and clinical findings discussed below point that knismesis, via its link to withdrawal behavior, may be closely associated to touch aversion and social touch avoidance. In this review we try to establish the importance of knismesis research in humans and to provide an initial research framework by integrating earlier findings on tickle and touch aversion with the recent advances in somatosensory neuroscience.

\section{The psychophysics and psychophysiology of knismesis}

Knismesis is produced by very light moving touch and produces elevated cortical arousal [12*]. For hairy skin this force is particularly low; we have been able to consistently elicit knismesis by a peacock feather brush that can transmit the vertical indentation force not exceeding $.05 \mathrm{~N}$. When stroking the forearm, this brush often did not touch the skin itself, just the skin hairs, but provided a very intense tickle sensation. Such light strokes do not induce tickle sensation in glabrous skin. One can take a fine feather or a grass blade and pull it very lightly along the skin of the inner forearm and the palm; the contrast between the forearm and the palm would normally be quite striking, and the sensation stemming from hairy skin stimulation would be clearly more arousing. Figure 1 a shows the difference in subjectively rated tickle intensity for knismesis-targeting very light touch and CT-optimal slow gentle touch applied to hairy and glabrous skin [12*]. It might seem surprising that our palms and soles, which are generally considered ticklish spots for gargalesis, are that insensitive to knismesis; this should probably be accounted for by the difference in hairy and glabrous skin mechanoreceptors and points to the difference in neural mechanisms underlying knismesis and gargalesis.

When Yngve Zotterman first discovered C-type low threshold touch afferents in 1939 [13*] using slow and gentle strokes, he hypothesized them to transmit tickle. Since then, the systems of unmyelinated low threshold mechanoreceptors he discovered, called c-low threshold mechanoreceptors (C-LTMR), were subsequently found in all mammals so far tested $\left[14^{*}, 15^{* *}\right]$ and in humans (CT-afferents) $\left[16,17^{* *}\right]$ where they are hypothesized to be responsible for conveying pleasant and rewarding properties of gentle touch, reinforcing social touch interactions and prosocial behavior. It should be noted that slow strokes applied to hairy skin may induce either knismesis (for forces below or around .05 N [12*]) or pleasant calming sensation (for forces between .2 to $.8 \mathrm{~N}$ conventionally used for the CT-targeting affective touch roughly equivalent to pressure of 200 to $800 \mathrm{~Pa}$ and above [18-20]).

Despite the earlier hypotheses, to us it seems unlikely that knismesis is transmitted via slow unmyelinated afferents because of the urgency associated to the knismesis sensation and corresponding to the evolutionary role of knismesis, which is similar to the role of the fast pain system [21] and provides an urge to immediately eliminate a possible threat. The putative knismesis-transmitting afferents therefore should be characterized by high or very high conduction velocities presumably above $5 \mathrm{~m} / \mathrm{s}$, sensitivity to very light touch or to hair deflection, and rapid adaptation, as evidenced by preferential response to dynamic touch. Considering this, we would suggest that for the hairy skin the putative 'knismesis system' is at least partly represented by a population of rapidly adapting low-threshold myelinated hair follicle $A \beta$ and/or $A \delta$ afferents (HFA) $\left[14^{*}, 22^{* *}\right]$. A sensation somewhat similar to knismesis but less intense may be elicited by moderate force self-stimulation of glabrous skin sites (i.e., palms and soles); it might be caused 
by a learned reinforcement mechanism similar to one hypothesized to enhance subjective pleasantness of slow gentle touch delivered to the glabrous skin regions where CT-afferents failed to be found [17**].

Considering the neural pathways of knismesis, it has been suggested earlier that tickle is transferred via spinothalamic tract (STT) transmitting input from unmyelinated fibers along with A $\delta$ afferent input [23], as tickle ceases when the spinothalamic tract is sectioned [24]. However, later findings show that in 56 of the 65 cordotomy patients knismesis sensations were intact [25*]; further studies are required to provide a more definitive answer whether knismesis is transmitted solely via the STT or through the dorsal column as well.

Many researchers comment on the similarity between knismesis and itch or even define knismesis as 'moving itch' [1,2,4*]. Indeed, subjects report both tickle and an itchy sensation after knismesis-targeted stimulation. There are, however, two important differences between knismesis and itch suggesting that they have different physiological background. Firstly, knismesis arrives fast while itch sensation builds up slowly and is relayed by slow unmyelinated afferents [26]. Secondly, from the standpoint of motivational systems, knismesis and itch are somewhat opposite, with itch stimulating approach behavior (the craving to scratch) and knismesis urging withdrawal response (withdrawal from the source of the sensation); this is further supported by a study revealing the opposite impact of tickle and itch on interpersonal distance $\left[27^{*}\right]$. It is possible to hypothesize that itch sensation follows knismesis as knismesis-conveying afferents may stimulate itch-related neurons at the level of dorsal horn interneurons that are currently believed to play an important role in integration of primary afferent input of different somatosensory modalities [15**]. Such a two-phase reaction, withdrawal followed by an urge to scratch, would provide a more reliable way to get rid of skin parasites or biting insects.

The perceived valence of a knismesis-related sensation depends heavily on the perceptual context and the ticklee's traits. Earlier studies $\left[1^{* *}, 4^{*}\right]$ pose that knismesis is entirely unpleasant and noxious. However, similarly to gargalesis, for some people knismesis can be linked to mixed emotions and pleasurable experience, particularly if you are in a mood for arousing sensations and the tickler is someone you like and trust $\left[3^{* *}\right]$. A study eliciting knismesis revealed that on average the participants tended to report their experience as slightly pleasant [12*]. Mechanical properties of the stimulus also influence the affective value of knismesis. Some stimuli, like slow strokes of a soft plump brush applied at forces of around .1 to $.2 \mathrm{~N}$, may elicit moderately intense knismesis and at the same time activate pleasureconveying low threshold CT afferents; such a stimulus would be normally assessed as pleasurable, slightly ticklish, and moderately arousing.

It seems consensual that tickle ratings of knismesis-inducing stimuli are particularly high for the lightest touch that can be perceived by hairy skin, as contrasted to light or moderately light touch $\left[3^{* *}, 12 *\right]$. While it is possible that knismesis afferents respond more vigorously to lower than to higher forces, we would suggest that the decrease of tickle intensity with force stems, at least partially, from the inhibiting input from other touch afferents. It has already been established that afferent inputs from different mechanoreceptive submodalities may modulate each other: CT-targeted gentle touch and pain stimuli of different origins can reduce pain level [28-30], and pressure inhibits vibration-like tingling perception [31]. We suggest that, in a similar manner, knismesis sensation putatively elicited by stimulation of rapidly adapting HFA is inhibited by activation of slower adapting myelinated afferents conveying pressure.

\section{Knismesis and pathological touch aversion}

Many people are averted by knismesis but there is a neurodevelopmental condition which is specifically linked to touch defensiveness and, particularly, to very light touch intolerance - autism spectrum disorder (ASD). General defensiveness to social and non-social touch sensations (e.g., clothing) is common in ASD (for review see $\left[32^{*}-34^{*}\right]$ ) but there are numerous anecdotal evidence reflecting experiences of occupational therapists $[35,36]$ and autistic people pointing that it is the lightest, tickle-eliciting touch that yields the most negative reaction. A detailed account is provided by Temple Grandin, a prominent autism 
self-advocate [ $\left.37^{* *}\right]$. She contrasts exciting effect of light touch to calming effect of deep pressure and suggests that light touch should be avoided when interacting with children with ASD. No systematic research of knismesis perception in autism has yet been performed but, if further confirmed, this intolerance of light touch corresponds well to current understanding of knismesis and autistic sensory perception. We suggest that autistic hyper-responsiveness to light touch stems from two essential properties of knismesis: its intrinsically arousing quality and its complex multifaceted nature. First, knismesis-eliciting stimuli are particular in the way that they are evolutionary meant to elicit elevated arousal $\left[3^{* *}, 4^{*}, 12^{*}\right]$. Many autistic individuals are characterized by hyper-arousal and general hyperresponsiveness to sensory stimuli [38,39], and the resulting effect of knismesis makes very light touch completely overwhelming for such an autistic person [37**]. Second, it has already been noted that light touch perception is shaped by the afferent input of different touch submodalities. A single gentle stroke to the forearm brings in afferent input from rapidly adapting $A \beta$ and A $\delta$ HFA accompanied by slowly adapting $A \beta$-innervated Merkel cells and followed by input from the CT-afferents, and the resulting percept requires a certain amount of sensory integration. The findings on sensory abnormalities in autism generally point to impairments in further processing of sensory input (reduced lateral inhibition, predictive coding deficits, and impaired sensory integration) $[33,39,40]$. Provine [3] notices that Grandin's descriptions of autistic response to light touch are strikingly similar to those of neurotypical subjects to highly aversive tickle; this can be a consequence of the less efficient inhibition of knismesis sensation by afferent input of other touch neurons.

It may seem plausible that light touch defensiveness observed in autism stems at least partly from knismesis hyper-responsiveness. But why should this be of particular importance, considering that the major challenges related to ASD are linked to social and communicative deficits? It was found that touch defensiveness, in addition to its adverse impacts on everyday life, is positively associated with social dysfunction in ASD adults [32*]. Touch defensiveness may decrease the frequency of caressing or playful touch interactions with family members affecting the trajectory of the developing social brain [41]. It has been previously hypothesized [17**] that social deficits in autism may be linked to the improper functioning or to insufficient stimulation of the C-tactile system that promotes prosocial behavior via oxytocin release [42]. Several papers reveal that social touch or touch-based interventions can enhance behavioral measures in autism $\left[37^{* *}, 43^{*}\right]$, decrease cortisol levels [44] or increase oxytocin release. It was found though, that CT-targeting touch leads to oxytocin release only when the touch is perceived as pleasant [12*]. It suggests that touch-based interactions would probably not yield any positive effect unless they bring positive emotional experience, confirming that forcefully administered touch interactions like holding therapy [45] would be useless if not harmful. When planning touch interventions, at least at initial stages, we suggest avoiding tickle sensations, particularly for individuals with high touch defensiveness. Possible touch-based interventions might utilize firmer, slower touch interactions highly predictable for autistic participants, either squeezing, as provided by Grandin's hug machine [43*], or stroking, massage-like, that would presumably provide greater activation of CT afferents. The data from smaller-scale studies suggest that such interventions may increase tolerance to lighter touch and turn it into a pleasurable experience $\left[37^{* *}, 43^{*}\right]$.

Other adverse emotional conditions have been linked to social touch avoidance, particularly the ones related to social anxiety and insecure attachment (for review see [46*]). Abnormal perception of affective touch has already been confirmed for anorexia nervosa [47] and attachment disorder [48]. It is yet unknown whether these abnormalities are related to knismesis but one may suggest that knismesiseliciting touch may be perceived as particularly unpleasant by individuals with prominent touch defensiveness, and this should be taken into account when considering touch-based or body-related interventions for these clinical populations as well. 


\section{Conclusions}

In this article we have tried to integrate the scarce existing information on knismesis (light tickle) to provide a conceptual framework for understanding the role of knismesis in affective touch (Figure 1b). We have hypothesized that knismesis is elicited by excitation of hair follicle afferents (HFA), induces itch via projections to itch neurons, and is strongly moderated by other mechanoreceptive submodalities, particularly by inputs from other low threshold $A \beta$ mechanoreceptors. Autistic touch defensiveness may reflect knismesis hyper-responsiveness and attenuated knismesis inhibition stemming from impaired integration of sensory inputs from different touch submodalities. It should be noted that the hypotheses laid out in this paper are still highly speculative due to the lack of data and should be challenged by further research using a range of behavioral and neurophysiological methods.

\section{Conflict of interest statement}

Nothing declared.

\section{Acknowledgements}

This study was supported by a Russian Government contract, project FZNM-2020-0005, "Transformation of human cognition and communication in the digital era". The authors would like to thank the anonymous reviewers for their insightful suggestions and express their deep gratitude to Prof. Francis McGlone who opened to us an exciting world of affective touch research, as he did to many others.

\section{References and recommended reading}

Papers of particular interest, published within the period of review, have been highlighted as:

* of special interest

** of outstanding interest

1. $\quad * *$ Hall GS, Allin A: The Psychology of Tickling, Laughing, and the Comic. Am J Psychol 1897, 9:1. The article that first describes two kinds of tickle, knismesis and gargalesis

2. Harris CR: The mystery of ticklish laughter . 1999, 344:1-7.

3. **Provine R: Laughter: a scientific investigation. Viking; 2000.

A book containing important considerations on human and non-human response to tickle

4. $\quad$ *Selden ST: Tickle. J Am Acad Dermatol 2004, 50:93-97.

One of few review papers paying substantial attention to knismesis

5. Leavens DA, Bard KA: Tickling. Curr Biol 2016, 26:R91-R93.

6. Ozhegov SI: Dictionary of the Russian Language. Firebird Pubns; 1989.

7. Merriam-Webster Inc.: Merriam-Webster Dictionary. 2016.

8. Panksepp J, Burgdorf J: "Laughing" rats and the evolutionary antecedents of human joy? Physiol Behav 2003, 79:533-547.

9. Cloutier S, LaFollette MR, Gaskill BN, Panksepp J, Newberry RC: Tickling, a technique for inducing positive affect when handling rats. J Vis Exp 2018, doi:10.3791/57190.

10. Davila Ross M, J Owren M, Zimmermann E: Reconstructing the Evolution of Laughter in Great Apes and Humans. Curr Biol 2009, 19:1106-1111. 
11. Kilteni K, Engeler P, Ehrsson HH: Efference Copy Is Necessary for the Attenuation of SelfGenerated Touch. iScience 2020, 23:100843.

12. *Portnova G V., Proskurnina E V., Sokolova S V., Skorokhodov I V., Varlamov AA: Perceived pleasantness of gentle touch in healthy individuals is related to salivary oxytocin response and EEG markers of arousal. Exp Brain Res 2020, doi:10.1007/s00221-020-05891-y.

Describes the psychophysical and psychophysiological response to knismesis and suggests that tickle mau play an important role in pathological touch defensiveness

13. *Zotterman Y: Touch, pain and tickling: an electro-physiological investigation on cutaneous sensory nerves. J Physiol 1939, 95:1-28

A seminal paper on unmielinated afferents that first suggested a neural basis for tickle

14. *Abraira VE, Ginty DD: The sensory neurons of touch. Neuron 2013, 79:618-639.

Summarises the current knowledge on skin nechanoreceptors in rodents

15. ${ }^{* *}$ Abraira VE, Kuehn ED, Chirila AM, Springel MW, Toliver AA, Zimmerman AL, Orefice LL, Boyle KA, Bai L, Song BJ, et al.: The Cellular and Synaptic Architecture of the Mechanosensory Dorsal Horn. Cell 2017, 168:295-310.e19.

Establishes the important role of sensory pre-processing ath the level of the dorsal horn

16. Vallbo $\AA \mathrm{B}$, Olausson $\mathrm{H}$, Wessberg J: Unmyelinated afferents constitute a second system coding tactile stimuli of the human hairy skin. J Neurophysiol 1999, 81:2753-2763.

17. $* *$ McGlone F, Wessberg J, Olausson H: Discriminative and Affective Touch: Sensing and Feeling. Neuron 2014, 82:737-755.

Establishes the role of affective CT afferents

18. Löken LS, Wessberg J, Morrison I, McGlone F, Olausson H: Coding of pleasant touch by unmyelinated afferents in humans. Nat Neurosci 2009, 12:547-548.

19. Haggarty CJ, Malinowski P, McGlone FP, Walker SC: Autistic traits modulate cortical responses to affective but not discriminative touch. Eur J Neurosci 2020, 51:1844-1855.

20. Croy I, Bierling A, Sailer U, Ackerley R: Individual Variability of Pleasantness Ratings to Stroking Touch Over Different Velocities. Neuroscience 2020, doi:10.1016/j.neuroscience.2020.03.030.

21. Nagi SS, Marshall AG, Makdani A, Jarocka E, Liljencrantz J, Ridderström M, Shaikh S, O'Neill F, Saade $D$, Donkervoort $S$, et al.: An ultrafast system for signaling mechanical pain in human skin. Sci Adv 2019, 5:eaaw1297.

22. **Vallbo $A B$, Olausson $\mathrm{H}$, Wessberg J, Kakuda $\mathrm{N}$ : Receptive field characteristics of tactile units with myelinated afferents in hairy skin of human subjects. J Physiol 1995, 483:783-795.

Describes types of myelinated afferents in human hairy skin

23. Al-Chalabi M, Reddy V, Gupta S: Neuroanatomy, Spinothalamic Tract. 2021.

24. Pritchard EA: The Clinical Significance of Variations in Tickle Sensibility. Proc R Soc Med 1933, 26:697-704.

25. *Nathan PW: Touch and surgical division of the anterior quadrant of the spinal cord. J Neurol Neurosurg Psychiatry 1990, 53:935-939.

Reveals that tickle may be intact in cordotomy patients

26. Hachisuka J, Chiang MC, Ross SE: Itch and neuropathic itch. Pain 2018, 159:603-609. 
27. * Okamura Y, Ura M: If I Feel Ticklish, I Will Keep My Distance, but If I Feel Itchy, I Will Approach You: Ticklish and Itchy Sensations Influence the Interpersonal Distance. Int J Psychol Stud 2018, 11:1.

Reveals an important difference between itch-related and tickle-related behavours

28. Habig K, Schänzer A, Schirner W, Lautenschläger G, Dassinger B, Olausson H, Birklein F, Gizewski $E R$, Krämer HH: Low threshold unmyelinated mechanoafferents can modulate pain. $B M C$ Neurol 2017, 17:184.

29. Liljencrantz J, Strigo I, Ellingsen DM, Krämer HH, Lundblad LC, Nagi SS, Leknes S, Olausson H: Slow brushing reduces heat pain in humans. Eur J Pain 2017, 21:1173-1185.

30. Taneja P, Olausson H, Trulsson M, Vase L, Svensson P, Baad-Hansen L: Modulation of experimental facial pain via somatosensory stimuli targeting sensations of different valence. $J$ Oral Rehabil 2020, 47:720-730.

31. Cataldo A, Hagura N, Hyder $Y$, Haggard P: Touch inhibits touch: sanshool-induced paradoxical tingling reveals perceptual interaction between somatosensory submodalities. Proc $R \operatorname{Soc} B$ Biol Sci 2021, 288:20202914.

32. *Mikkelsen M, Wodka EL, Mostofsky SH, Puts NAJ: Autism spectrum disorder in the scope of tactile processing. Dev Cogn Neurosci 2018, 29:140-150.

Summarises the current knowledge of autistic touch processing

33. * Thye MD, Bednarz HM, Herringshaw AJ, Sartin EB, Kana RK: The impact of atypical sensory processing on social impairments in autism spectrum disorder. Dev Cogn Neurosci 2018, 29:151-167.

A review linking sensory processing issues to the core social deficits of ASD

34. * Lundqvist L-O: Hyper-responsiveness to touch mediates social dysfunction in adults with autism spectrum disorders. Res Autism Spectr Disord 2015, 9:13-20.

A cohort study revealing a link between touch defensiveness and impaired social outcomes in ASD adults

35. Tubbs J: Creative Therapy for Children with Autism, ADD, and Asperger's: Using Artistic Creativity to Reach, Teach, and Touch Our Children. Square One; 2012.

36. Coyne P, Fullerton A: Supporting Individuals with Autism Spectrum Disorder in Recreation. Sagamore Publishing; 2014

37. ** Grandin T: Calming Effects of Deep Touch Pressure in Patients with Autistic Disorder, College Students, and Animals. J Child Adolesc Psychopharmacol 1992, 2:63-72.

An autistic person's view of light touch intolerance and calming effects of gentle pressure

38. Markram K, Markram H: The intense world theory - a unifying theory of the neurobiology of autism. Front Hum Neurosci 2010, 4:224.

39. Pellicano E, Burr D: When the world becomes "too real": A Bayesian explanation of autistic perception. Trends Cogn Sci 2012, 16:504-510.

40. Robertson CE, Baron-Cohen S: Sensory perception in autism. Nat Rev Neurosci 2017, 18:671684.

41. Cascio CJ, Moore D, Mcglone F: Developmental Cognitive Neuroscience Social touch and human development. Dev Cogn Neurosci 2019, 35:5-11. 
42. Walker SC, Trotter PD, Swaney WT, Marshall A, Mcglone FP: C-tactile afferents: Cutaneous mediators of oxytocin release during affiliative tactile interactions? Neuropeptides 2017, 64:27-38..

43. * Edelson SM, Edelson MG, Kerr DCR, Grandin T: Behavioral and physiological effects of deep pressure on children with autism: A pilot study evaluating the efficacy of grandins hug machine. Am J Occup Ther 1999, 53:145-152.

A small sample study showing calming effects of gentle pressure on autistic behaviour

44. Field T: Social touch, CT touch and massage therapy: A narrative review. Dev Rev 2019, 51:123-145.

45. Mercer J: International Concerns About Holding Therapy. Res Soc Work Pract 2014, 24:188191.

46. Keizer A, Heijman J, Dijkerman H: Do transdiagnostic factors influence affective touch perception in psychiatric populations? Curr Opin Behav Sci 2022, 43:in press.

47. Crucianelli L, Cardi V, Treasure J, Jenkinson PM, Fotopoulou A: The perception of affective touch in anorexia nervosa. Psychiatry Res 2016, 239:72-78.

48. Spitoni GF, Zingaretti P, Giovanardi G, Antonucci G, Galati G, Lingiardi V, Cruciani G, Titone G, Boccia M: Disorganized Attachment pattern affects the perception of Affective Touch. Sci Rep 2020, 10. 


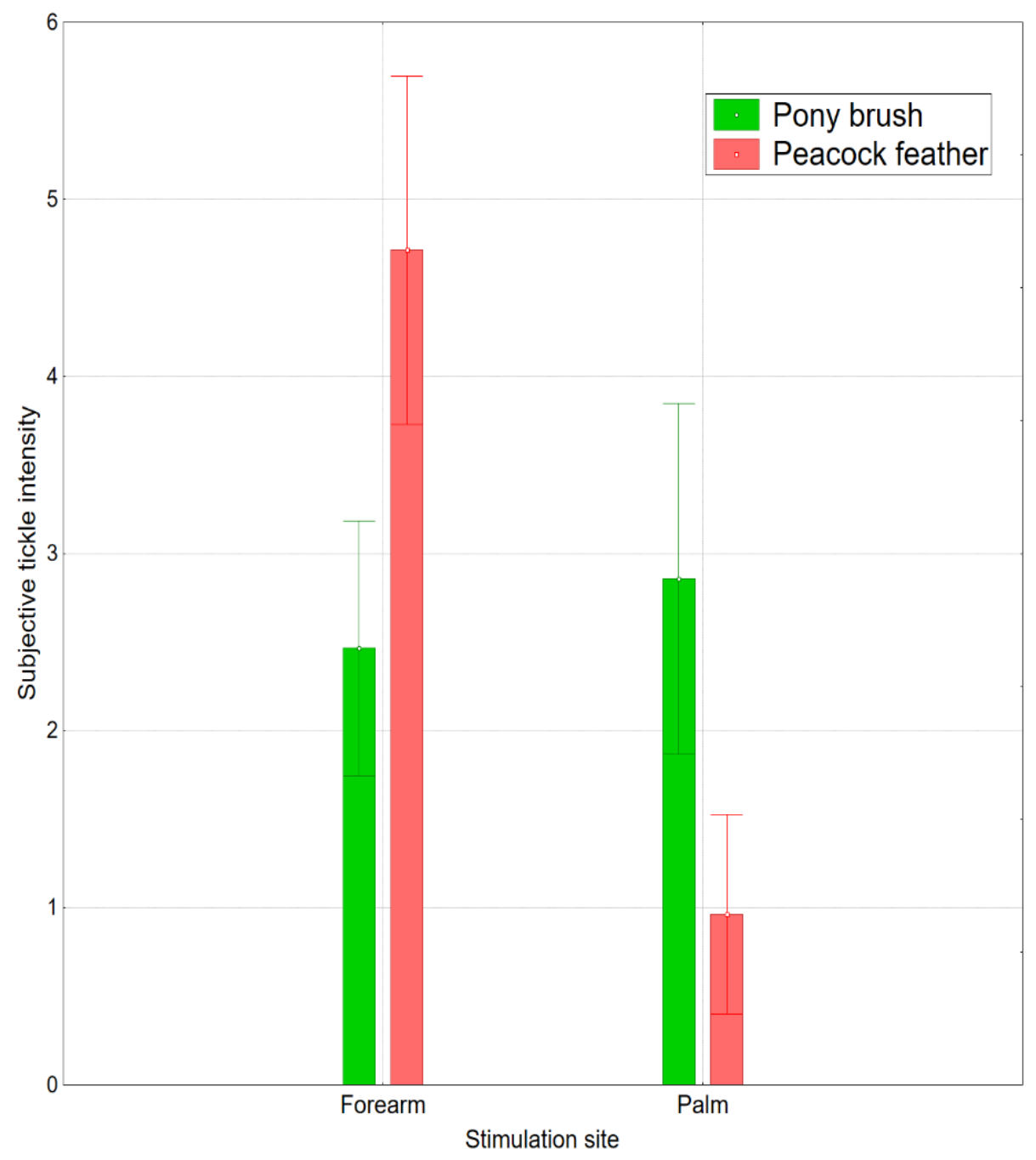

Figure 1a. The difference in subjective reports of tickle intensity of slow strokes delivered to the forearm and the palm with a peacock feather brush (force below $.05 \mathrm{~N}$ ) and pony brush ( $.5 \mathrm{~N}$ ). Based on data from Portnova et al. [14*]. 


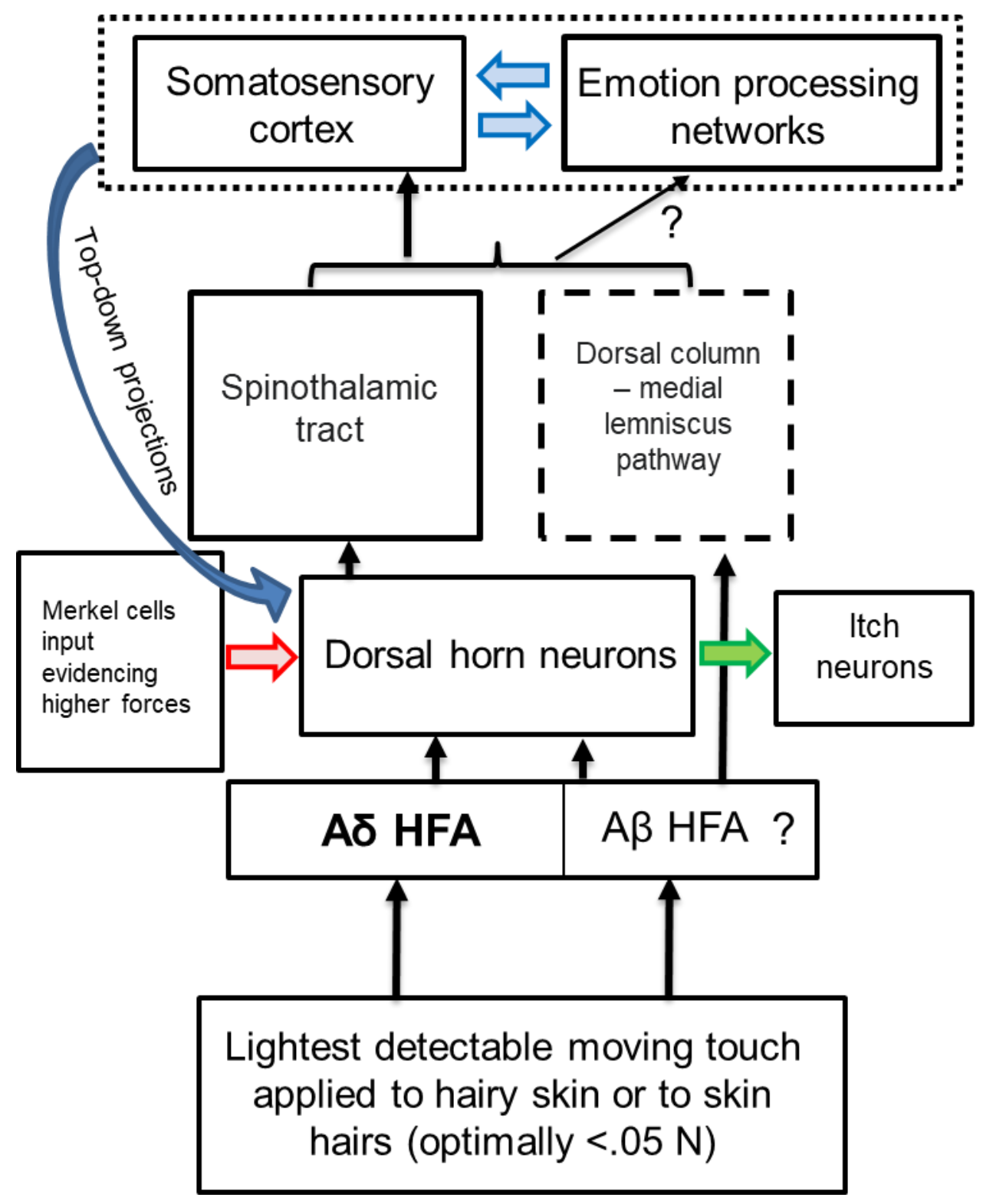

Figure 1b. A suggested theoretical model of physiological mechanisms underlying knismesis perception. 